Fabián V. del Valle

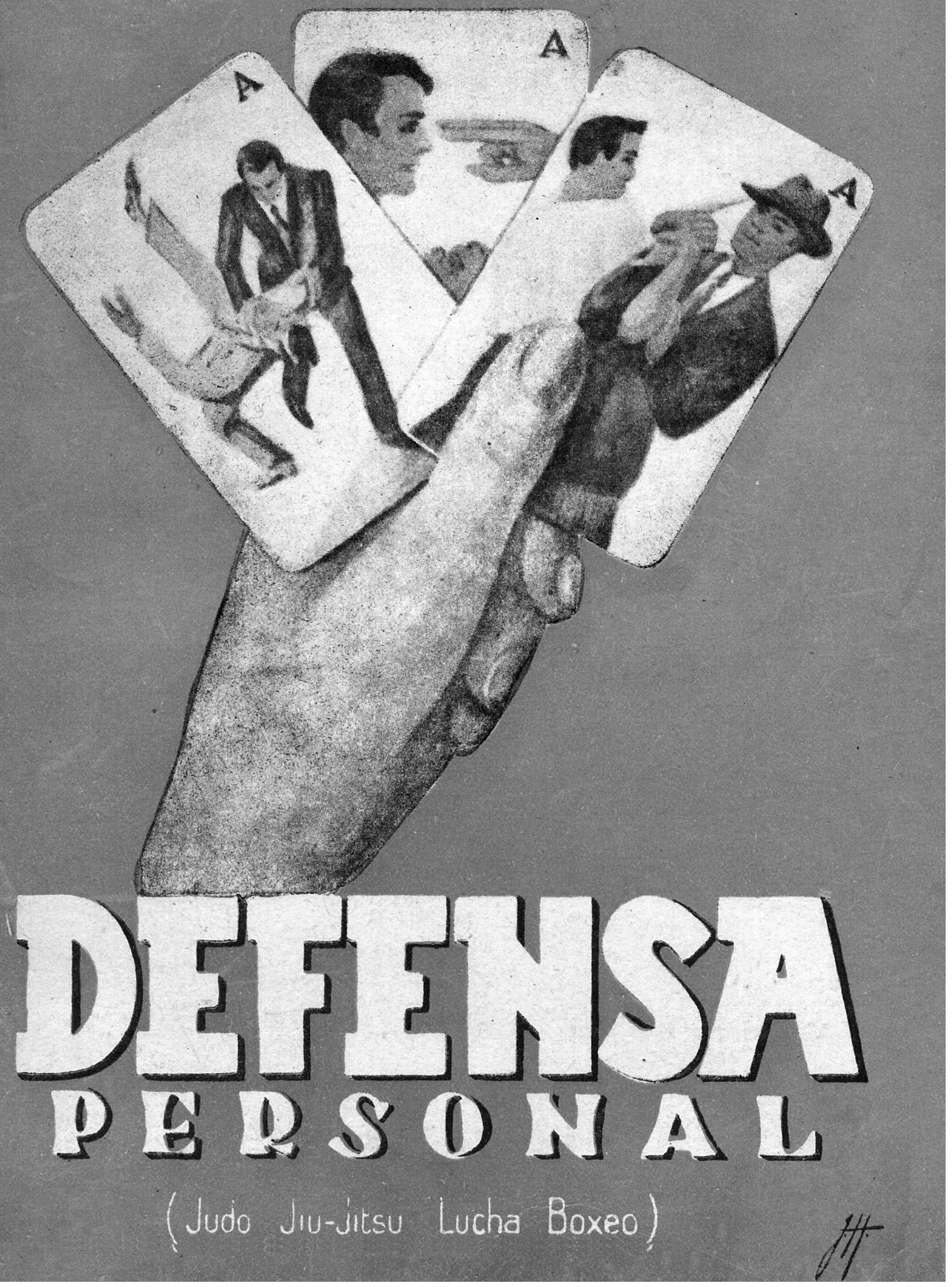




\title{
ESTUDIO BIBLIOMÉTRICO SOBRE LAS MONOGRAFÍAS DE ARTES MARCIALES PUBLICADAS EN ESPAÑA (1906-2006)
}

\author{
Mikel Pérez Gutiérrez y Carlos Gutiérrez García cure
}

\section{Resumen}

El presente estudio analiza desde una perspectiva bibliométrica las monografías sobre artes marciales publicadas en España entre 1906 y 2006. Partiendo del repertorio bibliográfico de Pérez y Gutiérrez (2008) se estudia el número total de monografías publicadas, número de monografías por materia, número de monografías por década, y número de monografías por materia y década. Los resultados muestran un número de 2.036 monografías ( 1.285 primeras ediciones), con un claro predominio de las artes marciales japonesas y chinas. Ocho materias superan el centenar de entradas (kárate, judo/ jujutsu, taijiquan, wu-shu/ kung fu, clásicos, qigong, filosofía, historia y educación, y aikido), revelándose dos modelos de evolución. El primer modelo, propio de las artes marciales japonesas consideradas en conjunto y de las artes marciales enfocadas fundamentalmente hacia los aspectos utilitarios y/o de rendimiento deportivo (judo/jujutsu, kárate y wu-shu/kung fu), evoluciona positivamente hasta los años ochenta, momento en el que se estabiliza y/o retrocede. El segundo modelo, propio de las artes marciales chinas consideradas en conjunto, y de aquellas artes marciales centradas principalmente en los aspectos higiénicos y/o espirituales (taijiquan, qigong, aikido), comienza un notable despegue en los años 90 hasta la actualidad. La explicación de estos modelos y de la propia evolución de la producción bibliográfica sobre artes marciales en España pone de relieve aspectos como el desarrollo de la sociedad española y de la práctica deportiva, de la producción bibliográfica, del hábito de lectura, o las influencias culturales de los países orientales en España.

\section{Introducción}

Los libros sobre artes marciales publicados en España ya han sobrepasado el siglo de historia, desde que en 1906 la parisina imprenta Librería de la Viuda de Bouret tradujese al español 100 coups de jiu-jitsu (coups et parades) [100 lances de Jiu-Jitsu (ataques y quites)], del francés Émile André (Gutiérrez, 2007). Desde entonces, la cantidad de libros sobre artes marciales editados en España, así como sus temáticas, han evolucionado irregularmente, adaptándose a las demandas informativas, modas y necesidades de la sociedad española. Hoy en día, estos cien años de historia arrojan una producción bibliográfica sobre artes marciales que supera ampliamente las 1.200 referencias, recopiladas en un reciente repertorio bibliográfico (Pérez y Gutiérrez, 2008). A partir de este repertorio, ha sido posible realizar un análisis bibliométrico sobre las monografías de artes marciales publicadas en España.

El análisis bibliométrico es una herramienta propia de las Ciencias de la Documentación que aplica métodos estadísticos a algunos de los datos encontrados en una referencia o registro bibliográfico, como puede ser la producción editorial o el análisis de las materias que más han interesado a los autores (López López, 1996; López Yepes,

PortadA DEL LIBRO

DEFENSA PERSONAL: JUDO, JIU-JITSU, LUCHA, BOXEO, ESCRITO POR FABIÁN Vicente del Valle Y EDITADO EN 1950 EN LA IMPRENTA DE FERNANDO Franco (MADRID). CON ESTA OBRA SE INAUGURABA LA SEGUNDA ÉPOCA DE LIBROS DE ARTES MARCIALES EN EsPaña.

Fotografía cortesía de Carlos Gutiérrez. 
2002). En este caso, el análisis bibliométrico permite revelar importantes indicadores sobre la evolución de las artes marciales en España, así como tomar conciencia de la situación y tendencias del mercado editorial español.

El presente artículo constituye, así, una investigación histórico-sociológica descriptiva e interpretativa sobre la producción editorial relativa a las artes marciales en España. Esperamos que este texto pueda aportar un poco más de luz sobre este campo de conocimiento, tanto sobre su historia como sobre el presente que viven las artes marciales en nuestra sociedad, y ayude a los estudiosos y profesionales del tema a adquirir una perspectiva más amplia sobre las orientaciones tomadas por las artes marciales.

\section{Aspectos metodológicos}

El objeto de este estudio lo constituyen los libros sobre artes marciales publicados en España y escritos en castellano o en alguna de las lenguas oficiales del territorio español. Estos criterios de lugar y lengua de publicación sirvieron para seleccionar aquellas obras que tienen como ámbito de distribución el territorio nacional, puesto que existen editoriales internacionales que editan libros en España pero que no se distribuyen en el mercado español, y su contabilización habría dado lugar a una estadística ficticia de la producción editorial para consumo interno. La única excepción a este criterio la constituyó la ya citada obra de Émile André (1906), publicada en París, pero que indudablemente tenía como ámbito de distribución España por estar publicada en castellano.

Respecto a la temática de las monografías, ésta debía dedicarse a las artes marciales de forma directa o como objeto de estudio de otras disciplinas, como puede ser el caso de la filosofía, la historia o la sociología de las artes marciales. Por tanto, la inclusión de una o varias artes marciales dentro de una monografía no significa su obligatoria inclusión dentro del campo de estudio, ya que, por ejemplo, las enciclopedias sobre deportes suelen dedicar algún capítulo a las artes marciales deportivizadas (judo, kárate, taekwondo, etc.) pero no conforman su temática principal.

Por otra parte, se siguió la definición sobre monografía dada por la norma UNE 50-104-19941 (AENOR, 1994) como criterio discriminador de las publicaciones que serían incluidas dentro del análisis. Además, se computaron las distintas ediciones de una misma obra como monografías y entradas distintas, puesto que informan sobre aquellas obras que han tenido una mayor aceptación y difusión a nivel tanto editorial como social al ser reeditadas.

En cuanto a la acotación temporal del estudio, éste comprende desde 1906, año de aparición de la primera obra de artes marciales publicada en castellano, hasta el año 2006, conformando así un siglo de publicaciones sobre artes marciales.

Para la creación del repertorio se consultaron las principales fuentes referenciales nacionales e internacionales, revisando para ello las bases de datos de la Biblioteca Nacional de España, Agencia Española del I.S.B.N. (International Standard Book Number), Catálogo Colectivo de las Bibliotecas Públicas del Estado y de la Red de Bibliotecas Universitarias (REBIUN), a nivel nacional; mientras que a nivel internacional se consultaron los catálogos de las bibliotecas nacionales del Reino Unido, Francia y Estados Unidos. Con un carácter secundario, se consultaron también diversos repertorios bibliográficos, los catálogos de las propias editoriales así como el portal Iberlibro para completar la información de las referencias más novedosas y antiguas respectivamente, y que aún no habían sido incluidas en las bases de datos principales.

La información extraída de estas fuentes fue recopilada en la ficha de descripción bibliográfica integrada en el programa gestor de referencias EndNote 9.0. Además de recopilar los elementos bibliográficos requeridos en la norma UNE 50-104-1994 sobre 
referencias bibliográficas, se añadieron otros elementos (materia, década y enlace) que facilitaron la recuperación de la información y la clasificación de cada obra para su posterior análisis. Parte de estos elementos se crearon a posteriori, tras el análisis del conjunto de obras recogidas. El criterio principal seguido en la división en materias de las obras fue el arte marcial tratado en cada obra o el campo de conocimiento desde el que se analizaba cada arte marcial. De esta forma, aparecieron 39 materias o subcategorías, que se podían agrupar en 16 categorías generales atendiendo al lugar de procedencia del arte marcial o la ciencia desde la que se abordaba esta temática (véase Tabla 1). Estos datos fueron exportados más tarde al programa Microsoft Excel 2003 para su tratamiento estadístico.

Por otra parte, y aunque el análisis bibliométrico puede centrarse en cuatro aspectos bien distintos como son la productividad, las materias, la colaboración y las citas (López López, 1996), el estudio se centró únicamente en los dos primeros por ser considerados los más relevantes para el propósito del estudio. De esta manera, el aspecto de productividad se refiere al número de monografías publicadas, mientras que el aspecto de materia indica cuáles han sido los temas que más han interesado a los autores. Estos aspectos dieron lugar a cuatro tipos de resultados:
1) Número total de monografías
2) Número de monografías por materia
3) Número de monografías por década, y
4) Número de monografías por materia y década.

Un último aspecto metodológico a comentar se refiere al análisis de la fiabilidad y de la adecuación de las fuentes utilizadas en el estudio (Aróstegui, 1995). El principal inconveniente en ambos sentidos fue la utilización de fuentes de información secundarias (bases de datos, catálogos y obras de referencia) en lugar de fuentes primarias (las propias monografías) para la obtención de la información, lo que conlleva, a su vez, una serie de limitaciones como son: a) la clasificación de las monografías en categorías inadecuadas, ya que ésta tuvo que ser hecha teniendo en cuenta el título de cada obra y la propia catalogación de los centros documentales de referencia; b) la información de ciertos elementos del registro bibliográfico, que suele ser diferente dependiendo de la base de datos que se consulte, probablemente debido a la aplicación de diferentes criterios de catalogación; y c) la posible pérdida de información, sobre todo de aquellas obras con una menor difusión. También hay que destacar como problemas de fiabilidad, aunque con un carácter secundario de importancia, la dificultad para triangular los datos de algunas de las obras más antiguas, que pueden estar registradas únicamente en una base de datos; la propia definición de monografía, ya que en algunos casos encontramos publicaciones de extensión inferior a las 30 páginas; y la falta de información respecto al número de ejemplares por edición, lo que significa dar la misma relevancia social a una edición de 100 ejemplares que a otra de 2.000 .

\section{Resultados y discusión}

El número total de monografías recopiladas ascendió a 2.036. Las primeras ediciones suman un total de 1.285 obras, siendo el resto reediciones (36.8\%). Las monografías se distribuyen en materias y décadas según muestra la Tabla 1. Como puede apreciarse, los países con una mayor influencia en nuestro país respecto al número de publicaciones sobre artes marciales son Japón y China, alcanzando un total de 850 y 545 monografías respectivamente, lo que significa el $68.5 \%$ del total. La interpretación del mundo editorial sobre artes marciales pasa necesariamente, por tanto, por el análisis pormenorizado de estos dos países. 
Tabla I: Distribución por materia y décadas de las monografías sobre artes marciales publicadas en España

\begin{tabular}{|c|c|c|c|c|c|c|c|c|}
\hline Categoria & Subcategoría & $\begin{array}{l}1950 \\
1959\end{array}$ & $\begin{array}{l}1960 \\
1969\end{array}$ & $\begin{array}{l}1970 \\
1979\end{array}$ & $\begin{array}{l}1980 \\
1989\end{array}$ & $\begin{array}{l}1990 \\
1999\end{array}$ & $\begin{array}{l}2000 \\
2006\end{array}$ & Total \\
\hline \multicolumn{2}{|c|}{ Obras anteriores a 1950} & \multicolumn{6}{|c|}{22} & 22 \\
\hline \multirow{10}{*}{$\begin{array}{l}\text { Japón } \\
(850)\end{array}$} & Aikido & & 3 & 9 & 11 & 41 & 37 & 101 \\
\hline & Judo y Jujutsu & 28 & 67 & 71 & 61 & 50 & 30 & 307 \\
\hline & Karate & & 18 & 95 & 91 & 49 & 66 & 319 \\
\hline & Kendo, Kenjutsu e laido & & & 2 & 5 & 7 & 4 & 18 \\
\hline & Ninjutsu & & & & 8 & 29 & 8 & 45 \\
\hline & Kyudo & & & & 1 & 1 & 1 & 3 \\
\hline & Taijutsu y Yawarajutsu & & & 1 & 5 & 2 & 1 & 9 \\
\hline & Sumo & & & & & & I & $\mathbf{I}$ \\
\hline & Kyusho y Kuatsu & & 3 & & I & 2 & 4 & 10 \\
\hline & Kobudo y otras armas japonesas & & & 12 & 20 & 4 & 1 & 37 \\
\hline \multirow{9}{*}{$\begin{array}{l}\text { China } \\
(545)\end{array}$} & Baguazhang & & & & 2 & 1 & 1 & 4 \\
\hline & Qigong $^{2}$ & & & & 10 & 47 & 58 & 115 \\
\hline & Taijiquan & & & 3 & 18 & 69 & 125 & 215 \\
\hline & Wu-Shu / Kung Fu & & 1 & 15 & 103 & 30 & 14 & 163 \\
\hline & Wing Chun & & & & 6 & 3 & 3 & 12 \\
\hline & Jeet Kune Do & & & & 2 & 7 & 7 & 16 \\
\hline & Choy Lee Fut & & & & 3 & 4 & & 7 \\
\hline & Xinyiquan y Yiquan & & & & 1 & 1 & 2 & 4 \\
\hline & Armas Chinas & & & 1 & 3 & 3 & 2 & 9 \\
\hline \multirow{3}{*}{$\begin{array}{l}\text { Corea } \\
(96)\end{array}$} & Taekwondo & & & 11 & 27 & 34 & 11 & 83 \\
\hline & Hapkido & & & 1 & 3 & 2 & 6 & 12 \\
\hline & Armas Coreanas & & & & 1 & & & $\mathbf{I}$ \\
\hline Filipinas (6) & Kali, Eskrima, Arnis & & & & 1 & 2 & 3 & 6 \\
\hline Tailandia (8) & Muay Thai & & & & 2 & 1 & 5 & 8 \\
\hline Malasia (y entorno) ( 1 ) & Pencak Silat & & & & 1 & & & $\mathbf{I}$ \\
\hline Vietnam (I) & Vovinam Viet Vo Dao & & & & & 1 & & $\mathbf{I}$ \\
\hline \multicolumn{2}{|c|}{$\operatorname{Otras}^{3}(43)$} & & & 1 & 18 & 11 & 13 & 43 \\
\hline \multicolumn{2}{|c|}{ Diccionarios y Enciclopedias (33) } & & & 2 & 16 & 10 & 5 & 33 \\
\hline \multicolumn{2}{|c|}{ Clásicos ${ }^{4}$ (128) } & & & 2 & 9 & 31 & 86 & 128 \\
\hline \multicolumn{2}{|c|}{ Filosofía, Historia, Educación ( 113 ) } & & 1 & 4 & 19 & 32 & 57 & 113 \\
\hline \multirow{3}{*}{$\begin{array}{c}\text { Defensa Personal } \\
\left(\begin{array}{l}(10)\end{array}\right.\end{array}$} & General & 4 & 15 & 13 & 12 & 19 & 7 & 70 \\
\hline & Femenina & & & & & 3 & 4 & 7 \\
\hline & Policial y Militar & 2 & 4 & & 3 & 7 & 17 & 33 \\
\hline \multicolumn{2}{|c|}{ Cine, Estrellas de Cine y Televisión (34) } & & & 7 & 8 & 13 & 6 & 34 \\
\hline \multicolumn{2}{|c|}{ Condición Física (13) } & & & 1 & 6 & 3 & 3 & 13 \\
\hline \multirow{3}{*}{$\begin{array}{c}\text { Miscelánea }^{5} \\
\text { (33) }\end{array}$} & General & & & & 3 & 4 & 1 & 8 \\
\hline & Chinas & & & & 1 & 11 & 9 & 21 \\
\hline & Japonesas & & & & 2 & 2 & & 4 \\
\hline \multicolumn{2}{|c|}{ Total } & 34 & 112 & 251 & 483 & 536 & 597 & 2036 \\
\hline
\end{tabular}


$\mathrm{Al}$ analizar el número de monografías por materia se observa, tanto en el conjunto de publicaciones sobre artes marciales japonesas como chinas, un grupo de tres materias que destacan sobre el resto al superar el centenar de obras. Dentro de Japón destacan particularmente el Kárate, el Judo/Jujutsu y el Aikido, mientras que en China despuntan el Taijiquan, el Wu-shu/Kung-fu y el Qigong. Estas materias forman parte de un conjunto de ocho categorías que sobrepasan el centenar de publicaciones (véase Gráfico 1), siendo el extremo opuesto otras con una representación mínima, como son el Sumo y el Kyudo de Japón, o el Baguazhang y el Xinyiquan de China. De esta manera se conforma un grupo de varias disciplinas que no superan las cinco publicaciones, reflejando un escaso interés editorial y, muy posiblemente, un interés social también escaso. En un punto intermedio, también con una considerable aportación, otras artes marciales como Taekwondo, Ninjutsu, Kick Boxing, Full Contact (categoría "Otras"), y Kobudo y otras armas japonesas. Dentro de las categorías no adscritas específicamente a un arte marcial destacan los Clásicos, Filosofía, Historia y Educación, y la Defensa Personal, reflejando el interés

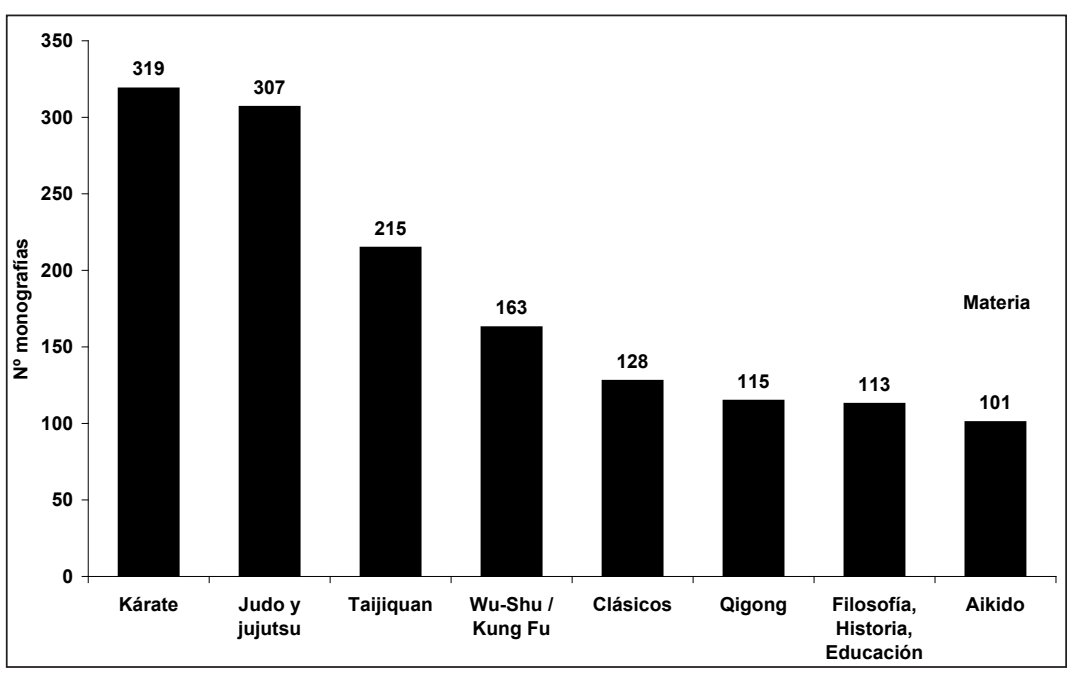
que han despertado estos aspectos tanto teóricos como aplicados en los consumidores de literatura marcial.

Aunque podría pensarse que el número de publicaciones de una materia se encuentra relacionado con la fecha de aparición de la primera obra de dicha materia -y en cierta medida así es-, esta relación no es general, ya que existen materias con una temprana fecha de aparición de su primera obra y un número reducido de publicaciones, caso del Baguazhang, en 1982, o el Kyudo, en 1988, pero que cuentan con una 4 y 3 publicaciones respectivamente; y en el extremo opuesto materias como el Jeet Kune Do y el Wing Chun que tienen una fecha de aparición similar a las anteriorres, 1984 y 1981 respectivamente, y con un número de publicaciones relevante, alcanzando las 16 y 12 monografías.

Esta relación entre fecha de aparición y número de publicaciones sí se constata en aquellas materias con una mayor producción bibliográfica. El Judo/Ju-jutsu tiene su época de implantación definitiva entre los años 1950 y 1965 (Gutiérrez, 2004), confirmando su relevancia a nivel nacional e internacional tras su integración en el programa de los JJ.OO. de Tokio 1964. Por su parte, el kárate crece en España al calor del judo, aprovechando el auge que estaba teniendo este arte marcial, e incluso dependiendo su práctica de la Federación Española de Judo. Por su parte, el Wushu/Kung Fu se implanta en España durante la década de los años 70 debido al gran impacto de las primeras películas sobre artes marciales protagonizadas por Bruce Lee y la famosa serie de televisión "Kung Fu" de David Carradine (año 1974-75) (Pérez Agustí 1995; Palmer, Palmer \& Meyers, 2003) lo que produjo un gran interés por este tipo de prácticas en la sociedad española. La aparición del Taijiquan también se produce en la década de los 70 y debe su popularidad al enfoque higiénico que presenta y que se desarrolló a partir de los años 80 .

Al realizar el análisis de la producción editorial por décadas se observa un crecimiento constante aunque irregular de la producción editorial sobre artes marciales, destacando dos momentos de inflexión. El primero de ellos se produce en la década

GRÁFICO 1: CONJUNTO DE CATEGORÍAS CON MAYOR NÚMERO DE PUBLICACIONES. 


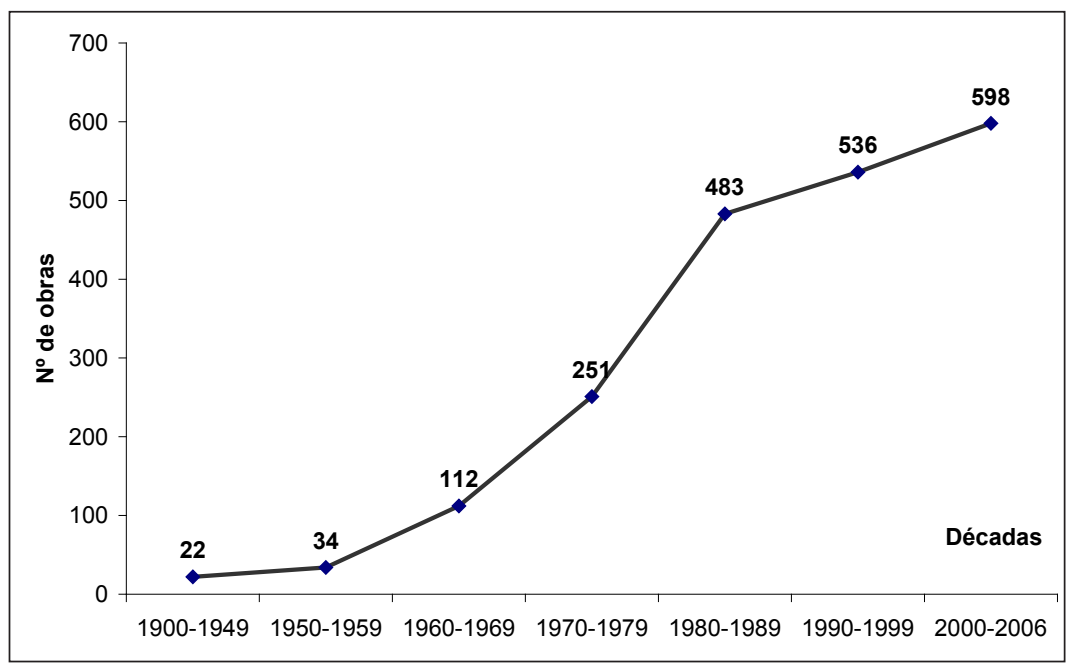

GRÁFICO 2: EVOLUCIÓN

POR DÉCADAS DE LA PRODUCCIÓN EDITORIAL SOBRE ARTES MARCIALES EN España. gable valor histórico (Gutiérrez, 2007). de los años 60, al doblarse la producción bibliográfica habida hasta la fecha, y el segundo en la década de los 80 , cuando se produce un gran crecimiento respecto a la década anterior (véase Gráfico 2). Estos momentos de inflexión darán lugar a dos modelos de evolución bibliográfica que se explicarán más adelante.

Desde una perspectiva histórica general, la primera mitad del s. XX (particularmente el periodo comprendido entre principios de siglo y la Primera Guerra Mundial) puede considerarse la primera etapa de introducción de las artes mar-

ciales en España. En consonancia con un sistema deportivo aún poco desarrollado, que salvo excepciones no lograba asegurar mínimamente la práctica de las artes marciales (en aquella época el jujutsu o jiu-jitsu) en los clubes y otras sociedades deportivas (Gutiérrez, 2004), la producción bibliográfica sobre artes marciales fue muy escasa, aunque de inne-

El resurgir de la economía española tras la Posguerra y la progresiva apertura del país acontecida desde los años 50 sería un importante acicate para el desarrollo de la práctica deportiva (Calatayud, 2002) y del mercado editorial. En el caso concreto de las artes marciales este proceso aperturista tendría una especial relevancia, ya que permitió la llegada a España de reputados profesores de artes marciales -en esta época profesores de judo-, así como los viajes de artistas marciales españoles al extranjero (Gutiérrez, 2004). Del mismo modo, la estabilización de la situación de Japón tras las desastrosas consecuencias sufridas por el país durante la II Guerra Mundial supondría el establecimiento de unas nuevas relaciones de influencia cultural recíproca entre Japón y Occidente, siendo las artes marciales una de las principales exportaciones culturales de Japón (Corcoran y Farkas, 1993). Tras la década de los 50, en la que se inicia la segunda época de implantación de las artes marciales en España, los años 60 supondrían su consolidación con hechos como la multiplicación de practicantes y profesores de judo, la difusión geográfica de la práctica, la creación de la Federación Española de Judo en 1965, o los comienzos de otras artes marciales en España como el kárate o el aikido. Esta consolidación se traduce en un primer punto de inflexión positivo en el desarrollo de la producción editorial sobre artes marciales.

Como consecuencia del resurgir de la economía española y del proceso modernizador que desde entonces experimenta el país, España llegaba pocas décadas después a un relativo Estado del Bienestar, con el logro de jornadas laborales más suaves, el aumento del poder adquisitivo, el desarrollo de los servicios avanzados, el aumento del tiempo de ocio, etc. (García Ferrando, 2001; Calatayud, 2002). Esta consecución y mejora del estado de bienestar tuvo sus repercusiones dentro del ámbito deportivo, destacando por un lado el fortalecimiento del deporte competición y el deporte espectáculo, apoyados fuertemente por los medios de comunicación, y por otro una gran diversificación de las practicas deportivas debido a cambios en los hábitos y motivaciones de los practicantes (García Ferrando, 2001). El deporte se convierte en un producto de consumo para las grandes masas a la vez que el mundo editorial impulsa y difunde el deporte dentro de ese proceso de mercantilización, convirtiéndolo en un mero producto que tiene que ser 
vendido. Todo ello tiene como consecuencia el aumento del número de practicantes de artes marciales y de artes marciales practicadas, así como el segundo punto de inflexión positivo en la producción editorial, durante la década de los 80 .

A partir de los años 90, y debido a los cambios en la composición sociodemográfica del país -mucha gente mayor, descenso del número de jóvenes y niños- y en los estilos de vida -estilo de vida urbano, mayor sedentarismo, hábitos alimenticios, ocio pasivo, etc.-, los practicantes tienden cada vez más hacia prácticas deportivas que fomentan la salud y el contacto con la naturaleza para contrarrestar los efectos de la vida cotidiana. Como constata García Ferrando (2001), existe una amplia mayoría de practicantes que hace deporte de forma recreativa, disminuyendo progresivamente el número de practicantes de deporte de competición. Este comportamiento también se observa en las artes marciales, y se traduce en aspectos como el descenso en el número de practicantes federados ${ }^{6}$. El cambio de mentalidad en los practicantes obliga a los profesionales de las artes marciales a transformar y enfatizar o añadir a sus prácticas otros valores como el deporte educativo, la recreación, la salud, la defensa personal, etc., e incluso otros contenidos acordes con estos nuevos valores. Este contexto también ha posibilitado que prácticas orientadas hacia la salud o el desarrollo espiritual o mental hayan ido afianzándose y ganando adeptos en la sociedad española, como es el caso del taijiquan, el qigong o el aikido, responsables en gran medida del aumento de la producción editorial?

Dentro de este contexto histórico cobra especial relevancia la propia evolución de la producción editorial española como marco general en el que ubicar la producción bibliográfica especializada en las artes marciales. Como muestra el Gráfico 3, se observa un crecimiento constante aunque irregular de la producción editorial desde comienzos del s. XX (Martínez, 2001), siguiendo la producción bibliográfica sobre artes marciales un patrón de evolución muy similar (Gráfico 2).

Este crecimiento de la producción bibliográfica sobre artes marciales también puede relacionarse con el incremento de los hábitos deportivos y de lectura de la población española, ambos constatados sucesivamente por García Ferrando (1990; 1997; 2001; 2006) desde 1975 y 1990 respectivamente (Gráfico 4). Sin embargo, y sobre la base de estos mismos estudios, se aprecia un constante descenso en el número de practicantes de las artes marciales, pasando de un 4\% (1990) a un 3,5\% (2000) y a un $2,2 \%$ (2005) respecto al número total de personas que practica algún deporte.
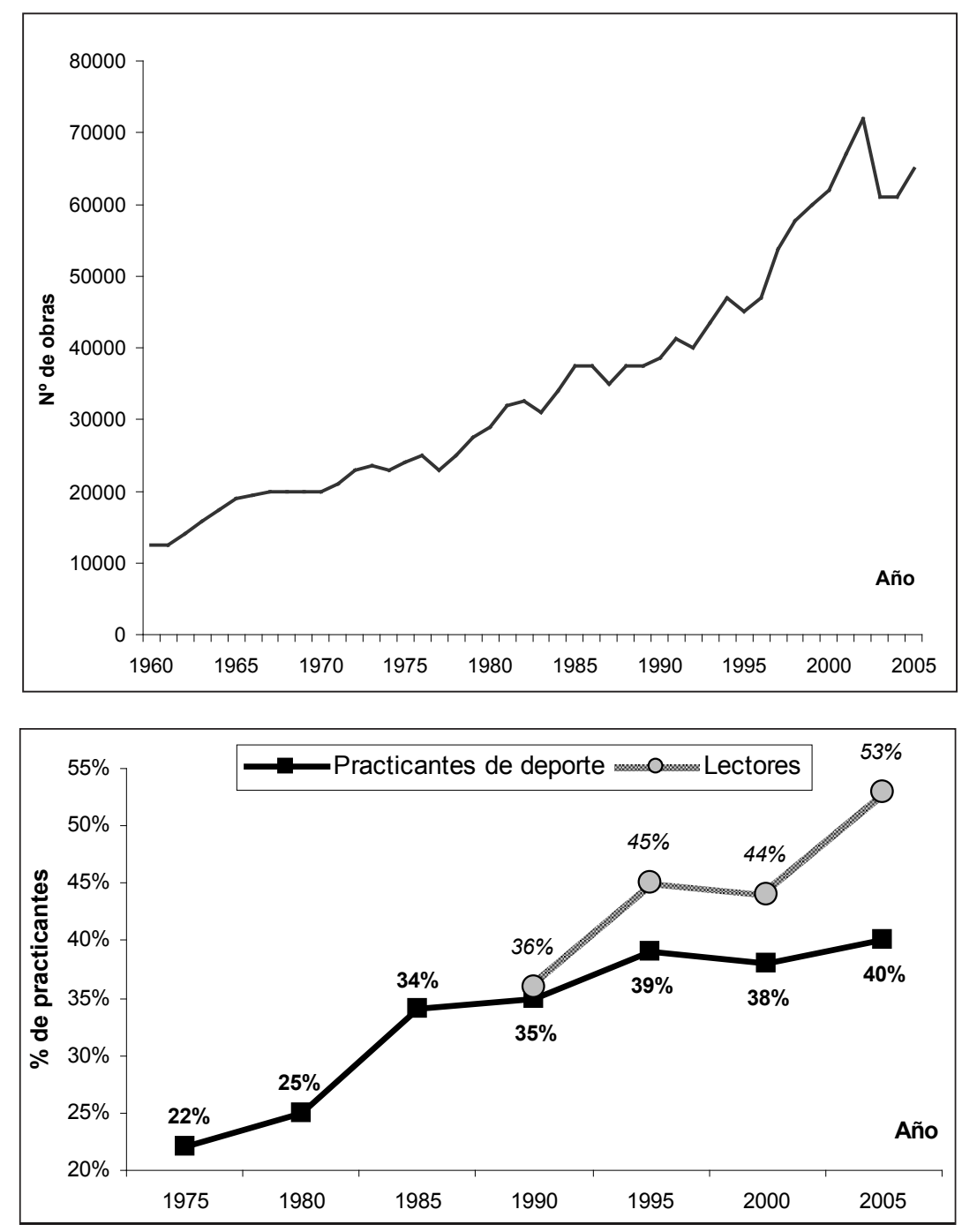

GRÁFICO 4: EVOLUCIÓN DE PRÁCTICA DEPORTIVA Y DEL HÁBITO DE LECTURA EN ESPAÑA. 
GrÁFICO 5: EVOLUCIÓN

BIBLIOGRÁFICA

DEL CONJUNTO DE ARTES

MARCIALES JAPONESAS Y

CHINAS.
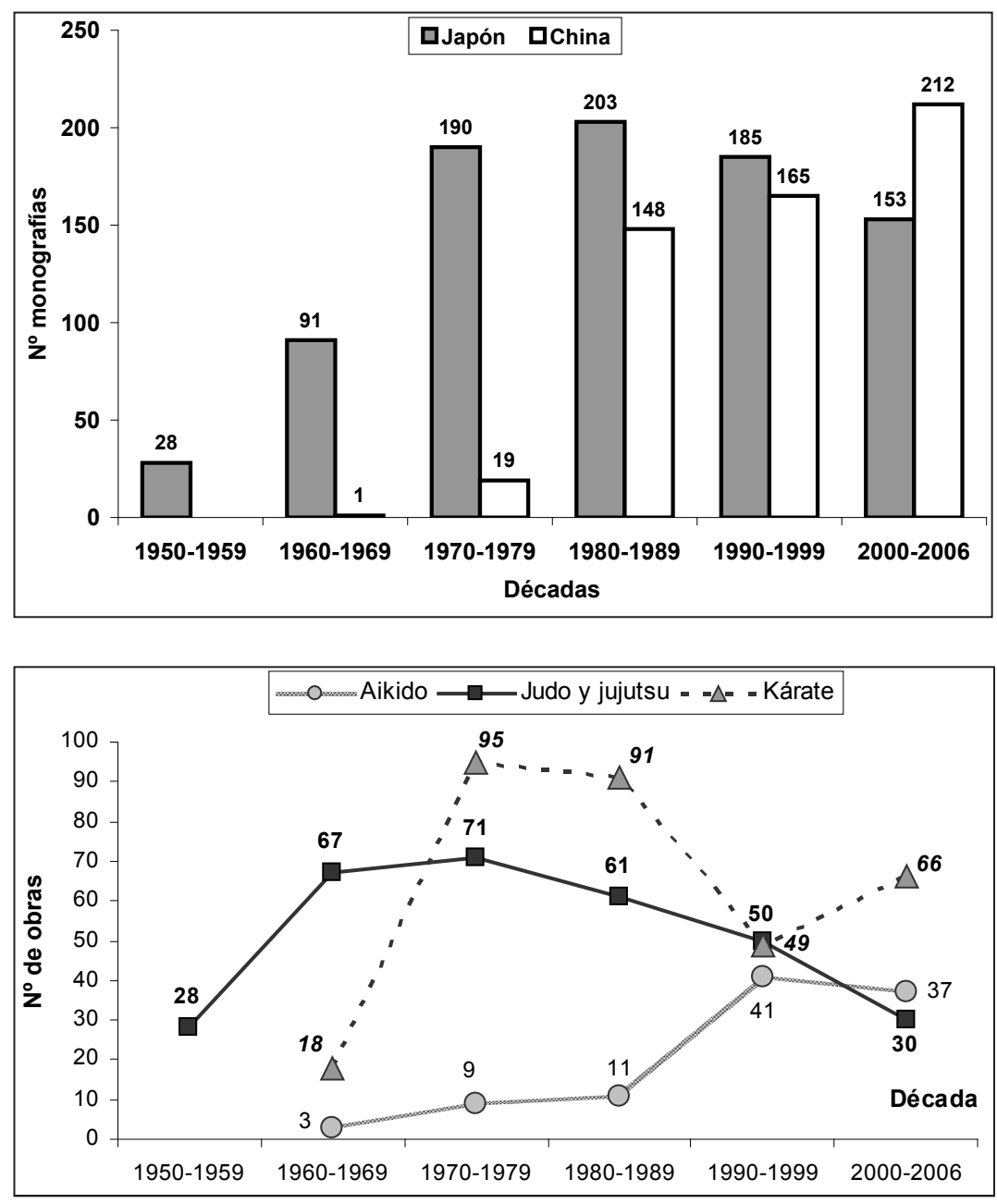

GrÁfico 6: EvOlución BIBLIOGRÁFICA DEL JUDO/ JUJUTSU, KÁRATE Y AIKIDO.
Con todo, y como ya han citado estudios anteriores (Gutiérrez y Pérez, 2008), los datos que ofrece García Ferrando tienen que ser analizados con precaución a los fines del presente estudio. Este autor incluye dentro de su estadística a los practicantes de deporte comprendidos entre los 15 y los 64 años -74 años en la encuesta del 2005-, lo que significa excluir a un gran número de practicantes de artes marciales que se encuentran por debajo de los quince años y que forman la base del deporte escolar. Así mismo, algunas de las prácticas que aquí se han definido como artes marciales, como es el caso del baguazhang, el qigong o el taijiquan, a menudo son practicadas bajo formas muy suaves que los propios practicantes pueden no llegar a conceptuar como artes marciales. De este modo, podrían ubicarse en otras categorías que comprende la clasificación de García Ferrando como son "Gimnasia de mantenimiento" u "Otros deportes". Por último, en la encuesta del 2005 García Ferrando considera una categoría de "Lucha, defensa personal", con un porcentaje del 1,6\%, que si se suma al $2,2 \%$ correspondiente a las artes marciales supondría un 3,8\%.

Particularizando el estudio de evolución por décadas en las diversas artes marciales, la primera gran etapa de evolución, entre las décadas de los 60 y los 80 , se debe al desarrollo de aquellas prácticas con un enfoque más utilitario y deportivo, respondiendo mayoritariamente las artes marciales japonesas a este modelo. El segundo momento de inflexión, a partir de la década de los 90, por el contrario, que se caracteriza por la aparición del deporte para todos y de otros nuevos enfoques más centrados en el desarrollo mental/espiritual e higiénico de sus practicantes, encaja mejor con la orientación principal tomada por las artes marciales chinas (véase Gráfico 5). Tampoco puede perderse de vista cómo la influencia de la cultura japonesa en España -y en general en todo Occidente- viene cediendo terreno a la influencia cultural china, entre cuyos principales bienes de exportación figuran también sus artes marciales.

La relación existente entre el enfoque tomado por las artes marciales y su evolución bibliográfica se constata al considerar las artes marciales chinas y japonesas más relevantes en cuanto a número de publicaciones. Como muestra el Gráfico 6, las artes marciales japonesas del judo/jujutsu y el kárate, que han orientado su desarrollo en un primer momento hacia el ámbito formativo y utilitario, y posteriormente hacia la competición deportiva, responden al primer modelo de evolución, mientras que el aikido, que se caracteriza por el rechazo de la competición deportiva y un mayor énfasis en los aspectos espirituales, responde al segundo modelo de evolución. Por su parte, las artes marciales chinas del taijiquan y el qigong, que enfatizan los aspectos saludables de 
la actividad física (relajación, respiración, desarrollo de la energía interna, etc.), responden al segundo modelo de evolución, mientras que el wu-shu/kung fu, centrado en aspectos utilitarios ligados a la eficiencia en el combate, responde al primer modelo (Gráfico 7). Asimismo, otras materias de corte más teórico, como son "Clásicos" y "Filosofía, Historia, Educación", dedicadas a los aspectos más históricos o filosóficos de las artes marciales, también se incluyen dentro del segundo modelo de evolución.

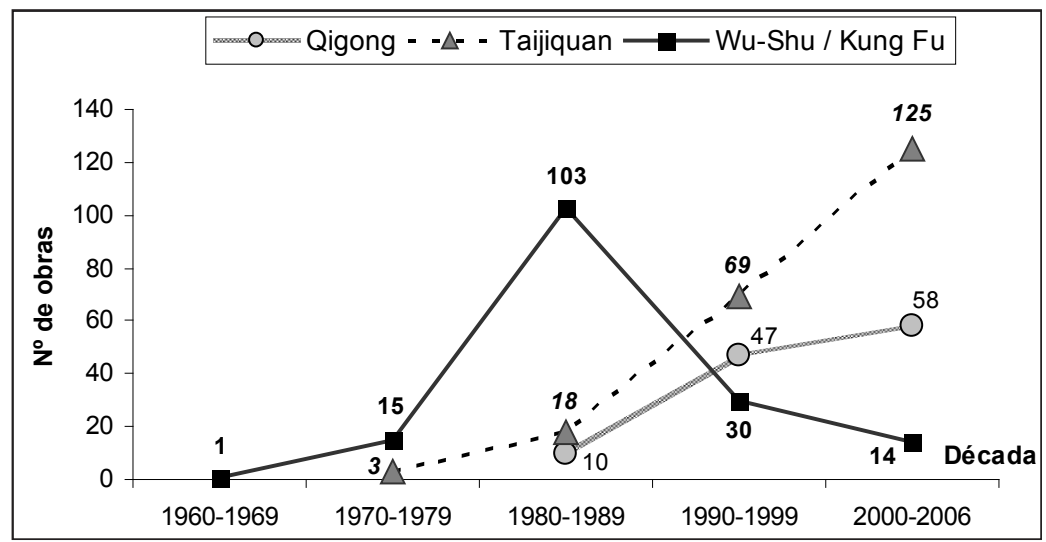

Este tipo de obras aparecen principalmente tras superarse una primera fase en la que predominan los manuales técnicos, centrados en los aspectos técnicos y/o tácticos de las GRÁFICO 7: EVOLUCIÓN prácticas marciales.

\section{Conclusiones}

La producción bibliográfica sobre artes marciales en España ha seguido un ritmo creciente desde principios del s. XX, y particularmente desde mediados de siglo cuando se inicia la implantación definitiva de las artes marciales en el territorio español. Esta evolución ha estado supeditada al devenir de la sociedad española a lo largo del s. XX, y particularmente a la evolución del mundo editorial y de la práctica deportiva. Existe una relación directa entre la evolución bibliográfica sobre artes marciales y el mundo editorial debido al logro de un estado de bienestar en el que la sociedad reclama y consume una mayor información acerca de las actividades deportivas que practica. Por otro lado, las artes marciales se han visto inmersas dentro de un proceso de diversificación y deportivación generalizado en el que se ha pasado de un deporte orientado fundamentalmente hacia la competición a un deporte recreativo, en el sentido más amplio de la palabra, orientado hacia la salud, el entretenimiento y como medio para contrarrestar el estilo de vida sedentario y estresante que prima en la sociedad actual.

El temprano enfoque formativo y utilitario de las artes marciales, concretamente de aquellas provenientes de Japón (judo/jujutsu y kárate principalmente), dio paso al modelo deportivo con el objeto de mejorar el estatus y número de practicantes de las diversas disciplinas marciales, lo cual se traduce en un notable incremento de publicaciones. A partir de la década de los 90 se transita hacia un fortalecimiento de aquellas prácticas con un enfoque espiritual/mental y/o higiénico, siendo las artes marciales chinas las que mejor se adaptan a estas perspectivas en la actualidad. Así, actualmente nos encontramos en una época de máximo esplendor en la publicación de monografías en este conjunto de artes marciales, mientras que las artes marciales más deportivizadas sufren una notable crisis de publicaciones que podría interpretarse sobre la base de su alejamiento respecto a los intereses y necesidades de la sociedad deportiva española.

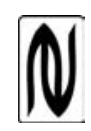




\section{NOTAS}

1 "Documento que no se publica en serie, es decir, que se presenta completo en un solo volumen o debe ser completado en un número finito de volúmenes" (p. 3).

2 La consideración del qigong como arte marcial es ciertamente problemática. Como señala Yang (1990), el qigong a) Tiene una vertiente marcial; b) Se asocia a determinadas artes marciales para mejorar su efectividad; y c) También se ha configurado como una práctica en sí misma encaminada al mantenimiento o recuperación de la salud. Debido a las manifestaciones a) y b) del qigong, se decidió incluir las monografías sobre esta práctica en el presente estudio.

3 Dentro de la categoría "Otras" se incluyen aquellas artes marciales que se conforman como la mezcla de otras de orígenes geográficos diversos, siendo el caso por ejemplo del full contact, nacido de la fusión del kárate con contacto y el boxeo (Perreca y Malora, 2003: 8-9); o el kickboxing, versión deportivizada del Muay Thai (Perreca y Malora, 2000: 11).

${ }^{4}$ La categoría "Clásicos" se refiere a obras que reeditan, amplían o comentan aquellas monografías que forman parte de una literatura de gran entidad debido a su antigüedad. Este es el caso por ejemplo de las distintas ediciones de El arte de la guerra, obra dedicada a la explicación de principios tácticos y estrategias militares aplicables a la guerra pero que constituye uno de los grandes clásicos de las artes marciales.

${ }^{5}$ La categoría "Miscelánea" incluye aquellas monografías que se dedican a varias artes marciales de diversos países (por ejemplo judo -Japón- y kung-fu -China-) o a prácticas marciales cuya aplicación puede referirse a varias artes marciales, como es el caso de los rompimientos.

${ }^{6}$ La suma y comparación del número de licencias expedidas por las federaciones vinculadas a las artes marciales (Judo y Deportes Asociados -Aikido, Jiu-jitsu, Kendo y wu-shu-, Kárate y Disciplinas Asociadas -Kenpo, Kung Fu y Tai Jitsu-, Taekwondo -en la que se incluye también el Hapkido- y Kickboxing) en los años 1990, 2000 y 2005, muestra un importante descenso del 28\% en el número de licencias entre 1990 y el 2000 (290.595 frente a 209.428), y un descenso mucho menor entre el 2000 y 2005 (204.699) (Consejo Superior de Deportes, 2007).

7 Nótese que hacemos referencia a orientaciones principales de las diversas artes marciales, lo cual no niega la existencia de otras formas de entender las mismas. Así, por ejemplo, el taijiquan en Occidente se ha conceptuado como un arte marcial "interno" o suave, más centrado en la salud que en el combate, en oposición a otros estilos marciales chinos "externos" o duros. La realidad, por el contrario, es que el taijiquan es también un efectivo y poderoso método de combate (Henning, 1997). Lo mismo sucede con cualquier otro arte marcial. Como bien apunta Rosa (2008: 39), "Hablar hoy en día de las prácticas de combate dual es hablar de una pluralidad de 'usos sociales' [...] podemos verificar algunos usos sociales: deportivos (internacionalización de las competiciones), profesionales (actividad remunerada), de integración (poblaciones consideradas de riesgo), higienistas (salud y desarrollo personal), de seguridad (preparación militar, fuerzas de seguridad), artísticos (estilos corporales o de vestuario), de gestión/administración (la incorporación de preceptos de las 'filosofías' marciales y del Extremo Oriente en los manuales de gestión) y turísticos (los locales de Shaolin en China, o el Aikikai en Tokio, entre otros). Bien entendido, que estos usos raramente existen en su estado 'puro'. Cada contexto concreto puede cruzarse y mezclarse con otros, en una gama prácticamente infinita de matices”.

\section{BIBLIOGRAFÍA}

AENOR (1994). Referencias bibliográficas: contenido, forma y estructura. UNE 50-104-1994. Madrid: AENOR.

André, Émile (1906). 100 lances de jiu-Jitsu. (Ataques y quites). París/México: Librería de la Viuda de Bouret. 
Aróstegui, Julio (1995). La investigación histórica: Teoría y Método. Barcelona: Crítica.

Calatayud Miquel, Francisco (2002). De la Gimnasia de Amorós al deporte de masas. Valencia: Ajuntament de València, Oficina de Publicaciones.

Consejo Superior de Deportes (2007). "Histórico de Licencias". Disponible en: http:// www.csd.mec.es/csd/asociaciones/1fedagclub/whistorico.xls. [Acceso 15/01/2008].

Corcoran, John y Farkas, Emil (1993). The Original Martial Arts Encyclopedia. TraditionsHystory-Pioneers. Los Ángeles: Pro-Action Publishing.

García Ferrando, Manuel (1990). Aspectos sociales del deporte: una reflexión sociológica. Madrid: Alianza Deporte.

García Ferrando, Manuel (1997). Los españoles y el deporte, 1980-1995. (Un estudio sobre comportamientos, actitudes y valores). Madrid: Consejo Superior de Deportes / Tirant Lo Blanch.

García Ferrando, Manuel (2001). Los españoles y el deporte: prácticas y comportamientos en la última década del siglo XX. Encuesta sobre los hábitos deportivos de los españoles, 2000. Madrid: Consejo Superior de Deportes.

García Ferrando, Manuel (2006). Posmodernidad y Deporte: Entre la Individualización y la Masificación. Disponible en: http://www.csd.mec.es/csd/sociedad/encuesta-dehabitos-deportivos/encuesta-de-habitos-deportivos/view. [Acceso 24/01/2008].

Gutiérrez García, Carlos (2004). Introducción y desarrollo del judo en España (De principios del s. XX a 1965): el proceso de implantación de un método educativo y de combate importado de Japón. [Tesis Doctoral]. León: Universidad de León, Vols. I y II.

Gutiérrez García, Carlos (2007). "Estudio de las primeras obras sobre artes marciales escritas en español". Revista de Artes Marciales Asiáticas, 2(1), 8-27.

Gutiérrez García, Carlos y Pérez Gutiérrez, Mikel (2008). "Evolución histórica de la producción de monografías sobre judo en España (1906-2006)”. Comunicación presentada en el I Congresso Cientifico Europeu de Judo, Lisboa, 10 de abril de 2008.

Henning, S. (1997). "Chinese boxing: The internal versus external school in the light of history and theory". Journal of Asian Martial Arts, 6(3), 10-19.

López López, Pedro (1996). Introducción a la bibliometría. Valencia: Promolibro.

López Yepes, José (2002). Manual de Ciencias de la Documentación. Madrid: Pirámide.

Martínez Alés, Rafael (2001). "El sector editorial español". ICE: Revista de economía, no 792, 109-123.

Palmer, Bill; Palmer, Karen; Meyers, Ric (2003). The Encyclopedia of martial arts movies. Lanhan (USA): Scarecrow Press.

Pérez Agustí, Adolfo (1995). 40 años del cine de artes marciales. Madrid: Adolfo Pérez Agustí.

Pérez Gutiérrez, Mikel y Gutiérrez García, Carlos (2008). 100 años de artes marciales: repertorio bibliográfico de monografías publicadas en España (1906-2006). León: Universidad de León.

Perreca, Giorgio y Malori, Daniele (2000). Kick Boxing: Preparación, técnicas, combate. Madrid: Tutor.

Perreca, Giorgio y Malori, Daniele (2003). Full Contact: Técnicas y preparación para el combate. Madrid: Tutor.

Rosa, Vítor (2008). "Las artes marciales y los deportes de combate en números: una mirada exploratoria sobre los datos numéricos o estadísticos en Portugal”. Revista de Artes Marciales Asiáticas, 3(2), 38-49

Yang, Shao-Ch'ing (1990). Jingang qigong. Ejercicios de respiración con movimientos de todo el cuerpo. ( $2^{\underline{a}}$ ed.). Madrid: Miraguano. 